\title{
Base-band involved integrative modeling for studying the transmission characteristics of wireless link in railway environment
}

\author{
Dawei Li $i^{1,2^{*}}$, Junhong Wang ${ }^{1,2}$, Meie Chen ${ }^{1,2}$, Zhan Zhang ${ }^{1,2}$ and Zheng Li $i^{1,2}$
}

\begin{abstract}
Base-band involved integrative modeling method (BIMM) is proposed for studying the transmission characteristics and bit error rate of the wireless communication, in which the transmitting and receiving antennas, wave propagation environment, and the modulation and demodulation modules are modeled and simulated integratively. Comparing with the conventional wave propagation method used in prediction of field coverage, BIMM is capable of taking the interaction between antenna and environment into consideration, and can give the time-domain waveforms of signals in different places of the wireless link, including those in the base-band modules. Therefore, the distortion reason of signal and in what place it happens can be found, and the bit error rate of the wireless communication can be analyzed. The BIMM in this paper is implemented by finite-difference time-domain (FDTD) method and is applied to the wireless link of railway communication. The effect of the electric spark generated by the power supplying network of the express train on the transmitting property and bit error rate is analyzed.
\end{abstract}

Keywords: Integrative modeling method; Wireless communication link; FDTD; Base-band signal; Railway environment

\section{Introduction}

With the rapid development and wide application of wireless communication, the prediction of the wave propagation and field coverage of the wireless link becomes important in characterizing the radio channel of the wireless communication, especially for the case of complicated environment, such as the railway environment. Usually, measurement is taken to get the field coverage of wireless communication systems, but measurement is expensive and time consuming and cannot give realistic results in some cases. For example, the results measured by an antenna in free space (normally used in measuring field strength) cannot reflect the realistic case of an express train system, in which the antenna is installed on top of the locomotive and is influenced by the locomotive body. Besides the measurement, numerical methods are also used in prediction of field coverage. There are basically two kinds of

\footnotetext{
* Correspondence: 11111001@bjtu.edu.cn

${ }^{1}$ The Key Laboratory of All Optical Network and Advanced

Telecommunication Network of Ministry of Education, No.3 Shangyuancun, Haidian District, Beijing, China, 100044

${ }^{2}$ The Institute of Lightwave Technology, Beijing Jiaotong University, No.3 Shangyuancun, Haidian District, Beijing, China, 100044
}

numerical methods used, one is the high-frequency method and the other is the full-wave method. The typical highfrequency method used is the ray-tracing method [1-4], which can give results with acceptable accuracy for simple and regular environments [3,4]. However, ray-tracing method is not suitable for analyzing the field coverage of the wireless link in complicated environments, so full-wave method is used in such environments. The typical fullwave method used is the finite-difference time-domain (FDTD) method [5,6], which has been used in studying the wave propagation in the micro-cell of mobile communication [7], between different floors of buildings [8], and in indoor environment [9]. It has also been combined with raytracing method to study the effect of wall on the indoor field coverage [10-12]. But these works did not consider the interaction between the transmitting/receiving antennas and environments and can only give the field distribution (field parameters). In our previous work, integrative modeling method (IMM) is proposed for characterizing the wireless link in complicated environments [13,14]. In IMM, the wireless link is decomposed into three parts, namely the transmitting antenna with its neighboring environment, 
the wave propagation environment between transmitting and receiving antennas, and the receiving antenna with its neighboring environment. These three parts can be integratively modeled and simulated by full-wave method only (small problem) or by hybrid methods involving full-wave methods and high-frequency methods (large problem). The difference between the IMM and other available methods is that IMM can not only give the field distribution in environment but also give the voltages/currents of input and output signals of the antennas (circuit parameters). With IMM, the effect of the environment on the output signal and the reason of signal distortion can be analyzed. In [13,14], FDTD is used to model and simulate the transmitting/receiving antennas together with their neighboring environments, and ray-tracing method is used to calculate the wave propagation in the rest environment.

However, IMM can only deal with the RF link of the wireless system and can give the reason of distortions of RF signals. But how these distortions affect the baseband signal? And how the environment influences the bit error rate of the wireless system? These are the motivations of this paper. In this paper, the modeling and analyzing of RF link (IMM) and base-band modules are combined together, and we call it the base-band involved integrative modeling method (BIMM). By this method, the time-domain waveform of signal at different places of the wireless link can be obtained, including those intermediate signal waveforms in base-band modules. So the reason of signal distortion and in what place it happens can be found, and the bit rate error of the complete wireless link can be studied. To our knowledge, there are no similar available works that can be used to analyze the relationship between distortion of base-band signal and environment.

In this paper, the wireless link of the railway communication is studied. Global system for mobile communications railway (GSM-R)-modulating signal, which is extensively used in express train, is adopted as the excitation signal. The detail description of the problem and the exciting signal involving the base-band information are given in Section 2. In Section 3, several examples are given to show the efficiency of the BIMM. Conclusions are drawn in Section 4.

\section{Problem description and method 2.1 Problem description}

The complete wireless communication link defined in this paper consists of modulation and demodulation modules, transmitting antenna, receiving antenna, and radio propagation environment, as shown in Figure 1.

Digital base-band signal modulates the carrier, and the modulated RF signal transmits to the space through the transmission antenna. When the wireless link environment is small enough, the transmitting and receiving antennas and the environment can be directly involved into the FDTD meshes by setting corresponding parameters at different mesh grids. The FDTD iteration will produce full-wave effects including the reflection, diffraction, and refraction between the antennas and environment. These effects will impact on the time-domain waveforms of the output signal of the receiving antenna. When the wireless link environment is too large to deal with by available computer resource, FDTD method could combine with ray-trace method to solve longrange propagation problem as mentioned in [13]. In this paper, we only consider the small-scale wireless link environment, so only FDTD method is used. The RF signal output from the receiving antenna is restituted through the demodulation module. By comparing the difference between input and output base-band signals, the transmission property of the whole wireless link and the error of bit rate can be studied.

\subsection{RF signal generation and base-band signal recover}

Actually, modulation mode of the base-band signal can be different for different systems. Gaussian-filtered minimum shift keying (GMSK) [15] is now taken as the main modulation mode in the GSM- $\mathrm{R}$ system for wireless communication in railway. The GMSK modulation can

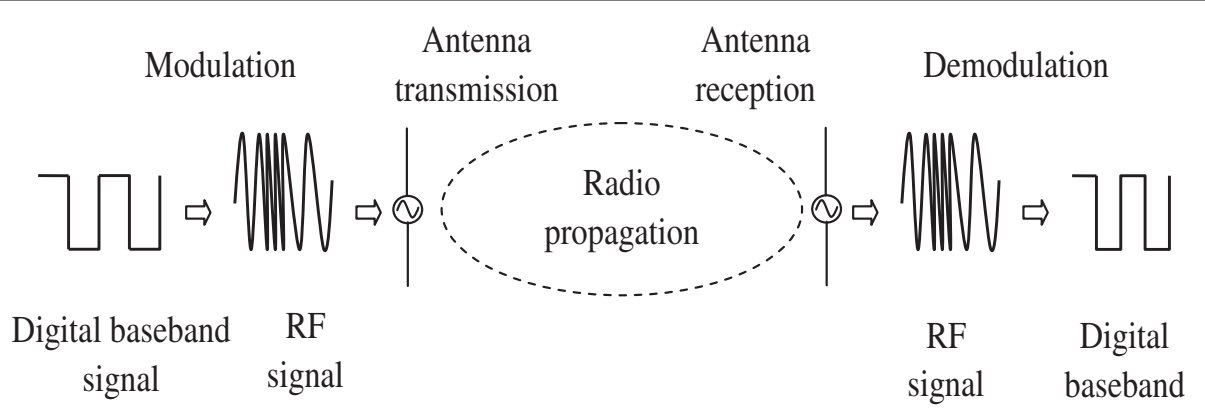

Figure 1 Schematic diagram of the wireless communication link. 


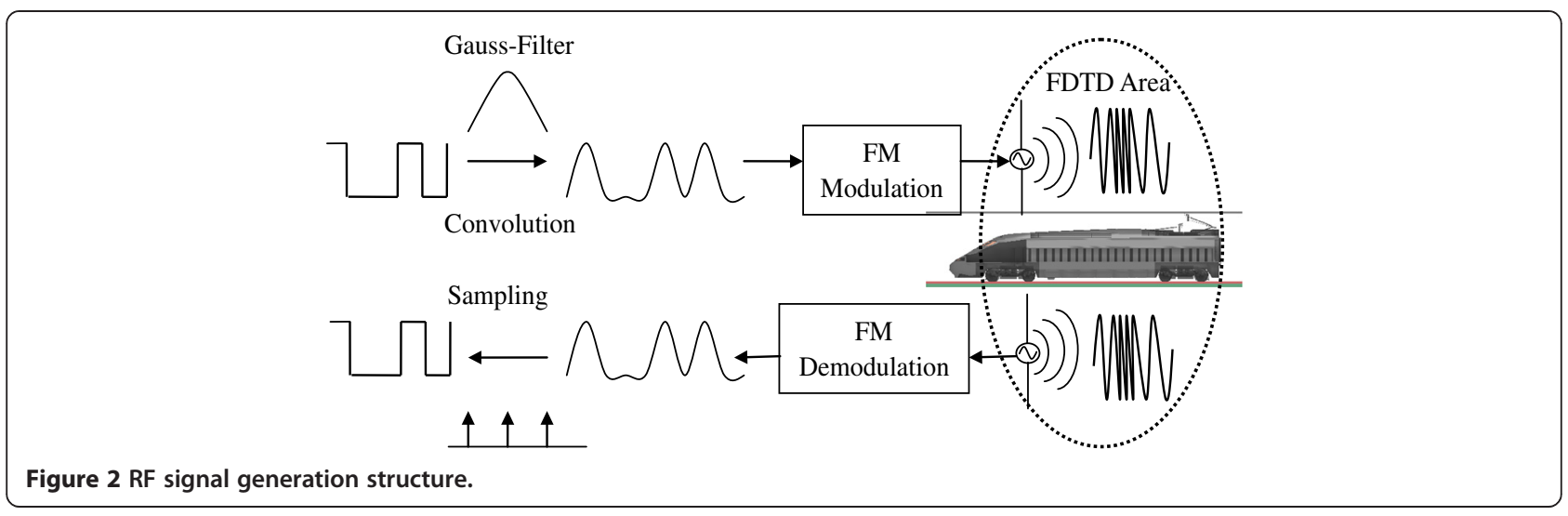

be realized by a pre-modulation low-pass filter (a general Gaussian low pass filter (LPF)) and a frequency modulation (FM) modulator. The Gaussian low pass filter can be represented by:

$$
\begin{aligned}
& h(t)=\exp \left(\frac{-t^{2}}{2 \delta^{2} T^{2}}\right) /(\sqrt{(2 \pi)} \cdot \delta T) \\
& \delta=\sqrt{\ln (2)} /(2 \pi B T)
\end{aligned}
$$

where $B$ is the bandwidth of the LPF and $T$ is the symbol period. $B T$ is a typical parameter of GMSK, and different $B T$ s present different correlations between adjacent symbols. Due to the limitation of computer resource, the rate of symbols in this paper is set to $5.4166 \times 10^{7} \mathrm{bps}$.
Further, the pulse response of the base-band signal is expressed by the following equation:

$$
g(t)=a_{i} \operatorname{rect}\left(\frac{t}{T}\right) * h(t) \quad\left(a_{i}=-1,1\right)
$$

The output signal from Gaussian LPF is modulated by FM algorithm by:

$$
x(t)=\sqrt{\frac{2 E_{c}}{T}} \cdot \cos \left(2 \pi f_{0} t+\int_{-\infty}^{t} g(\tau) d \tau+\phi_{0}\right)
$$

where $E_{c}$ is the energy of single bit, $f_{0}$ is the center frequency of carrier, and $\phi_{0}$ is the random phase of carrier. In this paper, the orthogonal frequency modulation is used and the center frequency is set to $900 \mathrm{MHz}$.
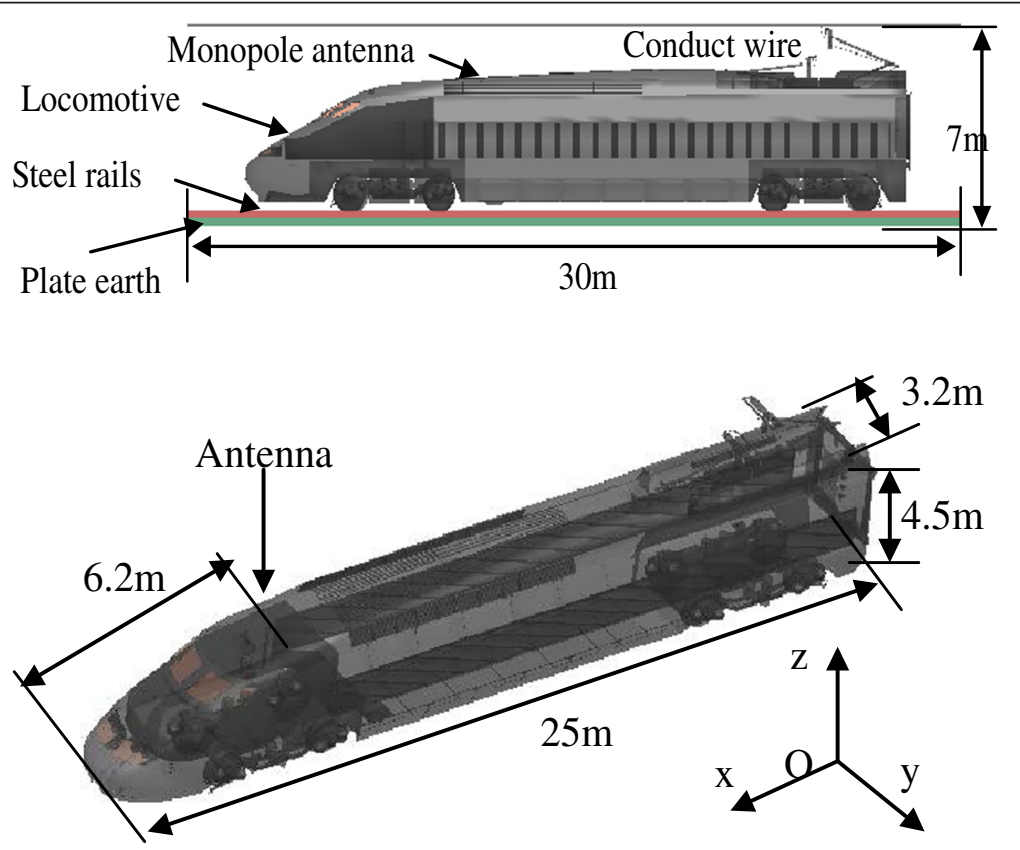

Figure 3 Modeling of locomotive and its environment. 
Table 1 Parameters of materials used in the model

\begin{tabular}{llll}
\hline Model name & $\boldsymbol{\varepsilon}_{\boldsymbol{r}}$ & $\boldsymbol{\mu}_{\boldsymbol{r}}$ & $\boldsymbol{\sigma}(\mathbf{S} / \mathbf{m})$ \\
\hline Locomotive body & 1.0 & 1.0 & $2.494 \times 10^{7}$ \\
Vehicle windows & 5.5 & 1.0 & 0.0 \\
Power supply wire & 1.0 & 1.0 & $2.494 \times 10^{7}$ \\
Steel rails & 1.0 & 1.0 & $1.1 \times 10^{6}$ \\
Earth plane & 14.0 & 1.0 & 0.01
\end{tabular}

The received RF signal is demodulated by the orthogonal coherent demodulation method. It is multiplied by the orthogonal carriers respectively first:

$$
\begin{array}{r}
x_{R x}(t) \cdot \cos \left(2 \pi f_{0} t\right)=\frac{1}{2}\left(\cos \left(2 \pi f_{0} t\right)+1\right) \cos \left(\int_{0}^{t} g(\tau) d \tau\right) \\
-\frac{1}{2} \sin \left(2 \pi f_{0} t\right) \sin \left(\int_{0}^{t} g(\tau) d \tau\right)
\end{array}
$$

$$
\begin{aligned}
x_{R x}(t) \cdot \sin \left(2 \pi f_{0} t\right)= & \frac{1}{2} \sin \left(2 \pi f_{0} t\right) \cos \left(\int_{0}^{t} g(\tau) d \tau\right) \\
& -\frac{1}{2}\left(1-\cos \left(2 \pi f_{0} t\right)\right) \sin \left(\int_{0}^{t} g(\tau) d \tau\right)
\end{aligned}
$$

and then passing through the LPF to extract the phase information in the modulated signal. After then, the signal is divided into two parts, that is, the quadrature signal and the in-phase signal:

$$
\begin{aligned}
& I(t)=-\frac{1}{2} \sin \left(\int_{0}^{t} g(\tau) d \tau\right) \\
& Q(t)=\frac{1}{2} \cos \left(\int_{0}^{t} g(\tau) d \tau\right)
\end{aligned}
$$

Finally, the base-band signal can be obtained by the two signals and their differentials using following equation:

$$
g(t)=-4\left(I^{\prime}(t)\right) Q(t)-I(t) Q^{\prime}(t)
$$

Digital signal can be recovered by sampling this signal. Figure 2 shows the modulation and demodulation process from base-band to base-band.

\subsection{Simulation scheme}

In this paper, a small scale wireless link environment is modeled using FDTD algorithm, which includes the transmitting and receiving antennas and the propagation environment. To further enhance the simulation speed, we adopted the parallel algorithm. Combining message passing interface (MPI) with FDTD to realize parallel computation has been proven to be an efficient way in improving the computing speed. The parallel algorithm of FDTD utilizes a one-cell overlap region to exchange the information between adjacent sub-domains, and only
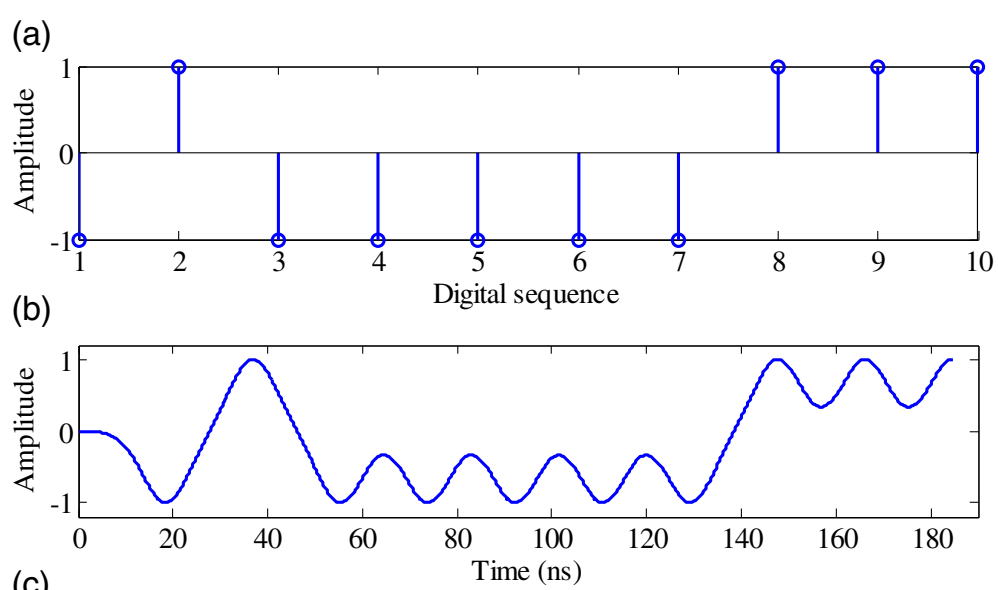

(c)

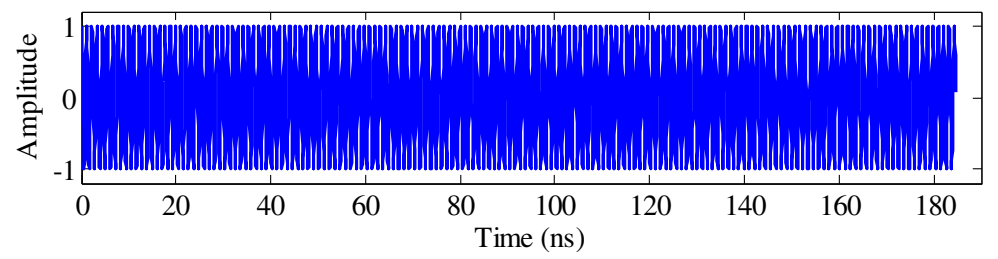

Figure 4 Generation of the base-band signal and RF signal. (a) shows the base-band digital data sequence. (b) shows the base-band signal after filtering. (c) shows the RF signal after modulation. 


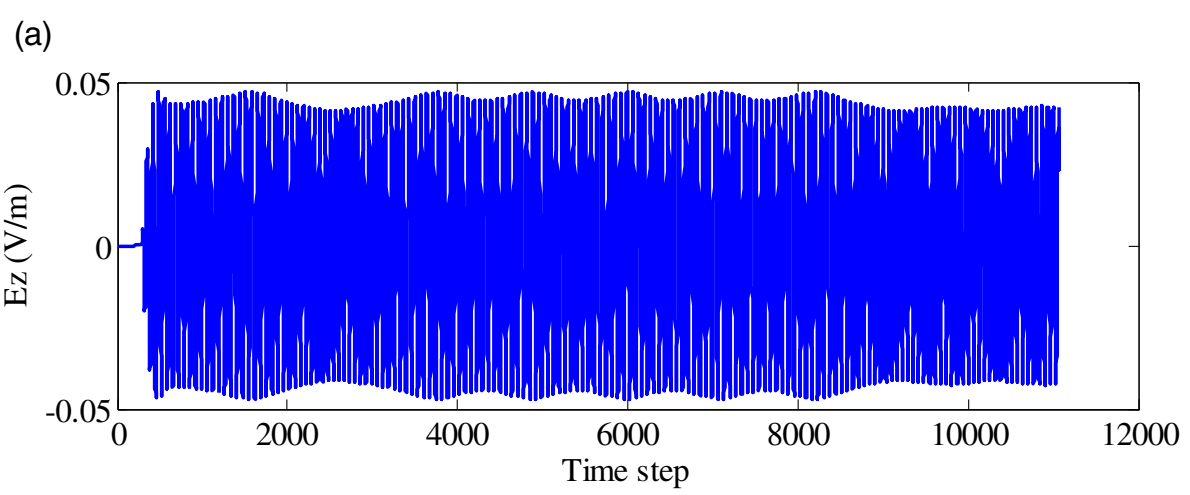

(b)

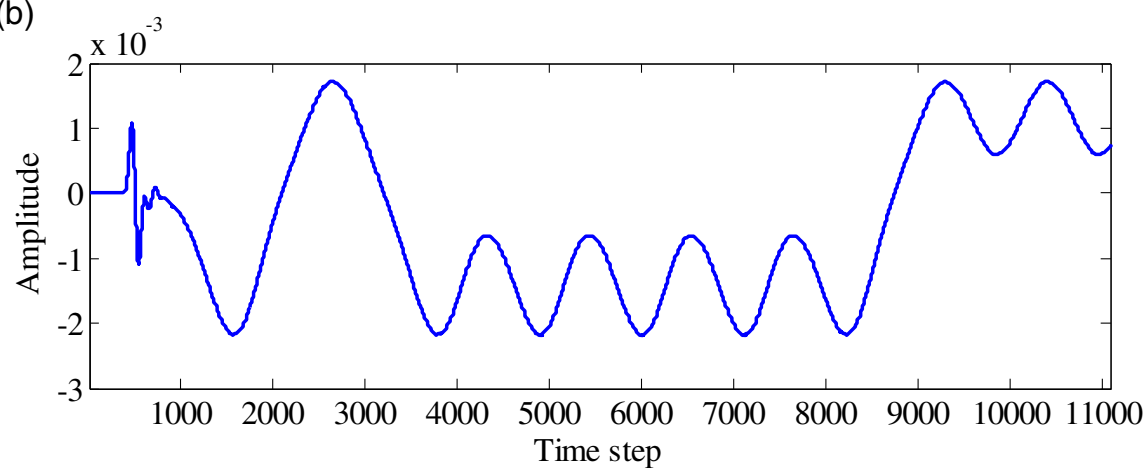

Figure $\mathbf{5}$ Received signal in free space. (a) and (b) show the RF signal received by the monopole antenna in free space (without locomotive) and the demodulated base-band signal, respectively.

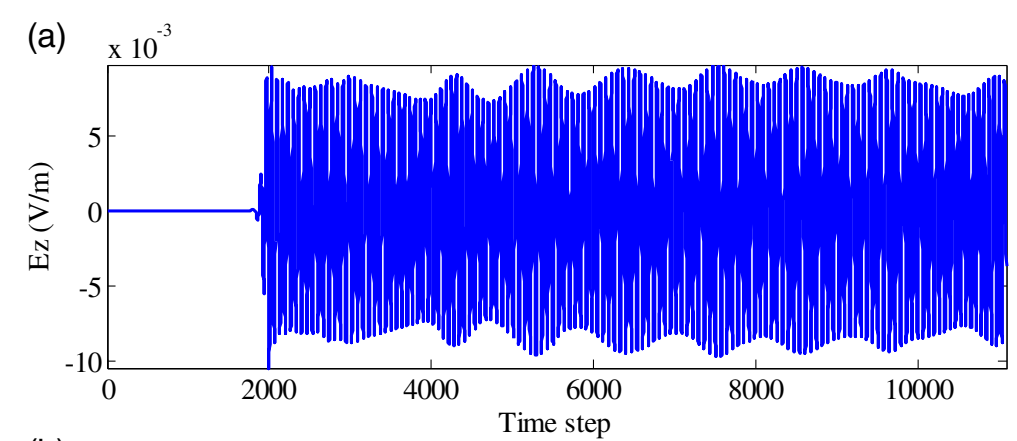

(b)

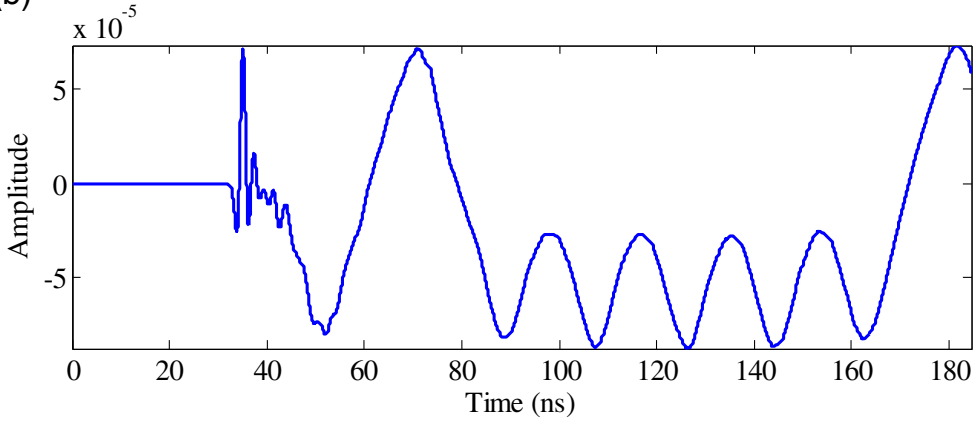

Figure 6 Received signal by locomotive antenna. (a) and (b) show the RF signal received by locomotive antenna and the demodulated base-band signal. 
the tangential magnetic fields are exchanged at each time step [16].

After all the parameters are set, the base-band signal is modulated into RF signal which excites the transmission antenna and starts the FDTD computation. The receiving antenna output the voltage amplitude in each time step of FDTD iteration for post-processing. This output RF signal involves the environment effect, such as the multi-path effect and the interference between antennas and nearby materials, and the demodulated base-band signal also involves the environment effect. Using this base-band-involved integrative modeling method, the entire wireless communication link can be simulated accurately and the reason of bit error can be found.

\section{Implementation and results}

\subsection{Modeling of antennas and environment}

In order to show the capability of BIMM in analysis of base-band signal transmission and bit rate error of wireless system, an example is given, which involves a locomotive with antenna mounted on top, two steel rails, earth plane, and a conducting wire over the locomotive for power supply. Figure 3 shows the detail description of these models drawn in CAD. The electromagnetic parameters for these models are listed in Table 1. In this example, a dipole antenna is used as the transmitting antenna, and a monopole antenna mounted on the top of locomotive is used as the receiving antenna. These two antennas are located not far away, so they are involved
Table 2 Parameters of the electromagnetic spark used in this paper

\begin{tabular}{ll}
\hline Parameters & Values \\
\hline$U_{0}^{p}$ & $2.962 \times 10^{4} \mathrm{~V}$ \\
$t_{0}^{p}$ & $4.5 \mathrm{~ns}$ \\
$f_{0}^{p}$ & $500 \mathrm{MHz}$ \\
$T_{0}^{p}$ & $4.0 \mathrm{~ns}$ \\
\hline
\end{tabular}

into the FDTD computational domain together with the environment.

The center frequency of the antennas is set to $900 \mathrm{MHz}$, and the frequency band covers the signal bandwidth. The length and cross section of the transmitting half-wavelength dipole are 150 and $25 \times 25 \mathrm{~mm}$, respectively, and the feeding gap between two arms of the dipole is $10 \mathrm{~mm}$. The length and cross section of the monopole mounted on the locomotive top is 70 and $25 \times 25 \mathrm{~mm}$, respectively, and the feeding gap between locomotive top plane and monopole is $10 \mathrm{~mm}$. The dipole antenna is located $1 \mathrm{~m}$ above the locomotive and with a distance of $14.3 \mathrm{~m}$ (in the rear of the locomotive) to the monopole antenna. Non-uniform grid technique and parallel FDTD algorithm are utilized. The general grid size of FDTD is set to 30,30 , and $30 \mathrm{~mm}$ in the $x$, $y, z$ directions, respectively, while the non-uniform grid size in the antenna and nearby region is set to $12.5,12.5$, and $10 \mathrm{~mm}$, respectively. The locomotive is located along the $x$-direction. The surrounding environment is

\section{(a)}

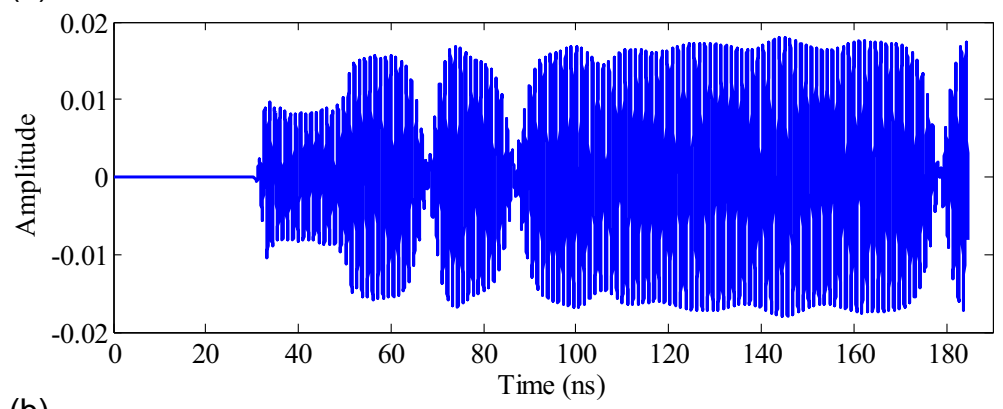

(b)

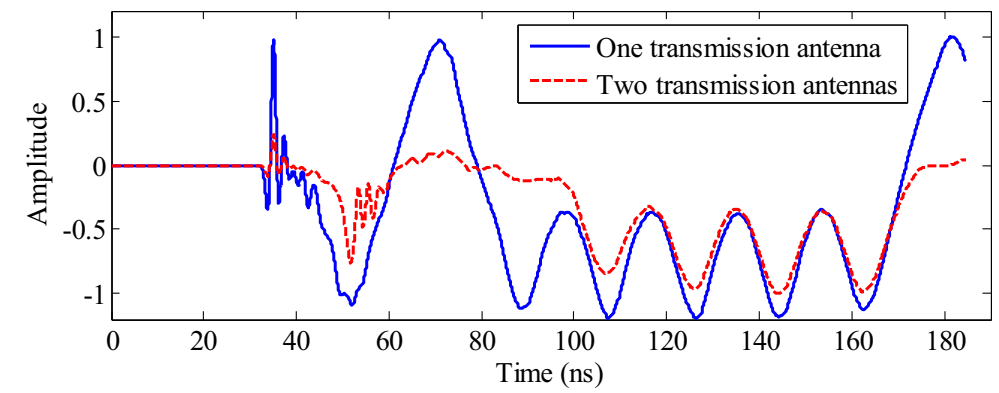

Figure 7 Received signal. (a) and (b) show the received RF signal and demodulated base-band signal for the case of two dipoles radiating simultaneously at different places. 


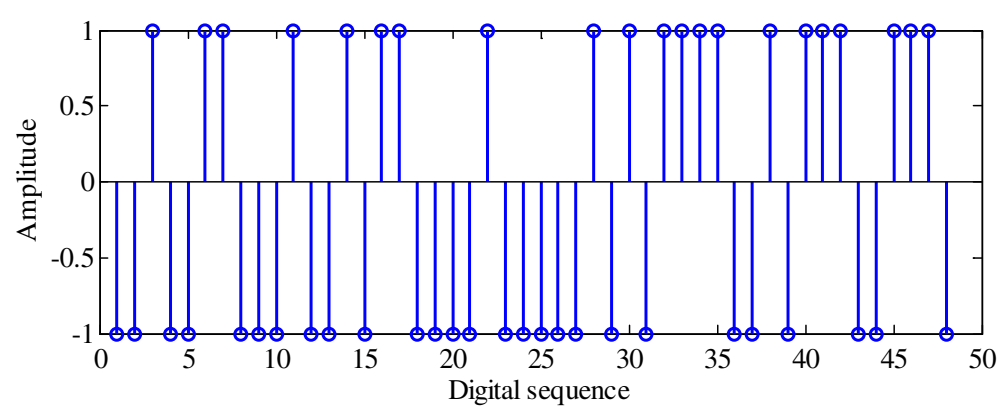

Figure 8 Input digital signal of the simulation.

divided into $1,109 \times 218 \times 312 \Delta S$ grids. The feeding gap of the locomotive antenna is located at the coordinates of $(755,105,190 \Delta S)$, and the feed gap of the transmitting dipole is located at $(105,172,254 \Delta S) . \Delta t$, the time step of FDTD, equals $16.667 \mathrm{ps}$, and the total computation time steps are set to 11,070 .

\subsection{Impact of environment on transmission signal}

In this paper, a sequence of data is modulated by the GMSK scheme. The data rate of GMSK used in this paper is $5.4166 \times 10^{7} \mathrm{bps}$, and parameter $B T$ in Equation 2 is set to 0.5 . The sequence of the digital data and the waveform of the base-band signal after filtering are shown in Figure 4.

In order to see the impact of the antenna itself on the transmission of RF signal, the receiving monopole antenna is placed individually in free space (without locomotive and environment) first, and the received signal is shown in Figure 5. From this figure, we can see that the envelope of the received RF signal is slightly undulated as shown in Figure $5 \mathrm{a}$, but this does not have much influence on the demodulated base-band signal as shown in Figure 5b. Therefore, the effect of antenna can be ignored.

The monopole is then put on the locomotive, and the received signal with the effect of the locomotive and environment is shown in Figure 6. This time, a larger fluctuation of amplitude of the RF signal can be observed. But the demodulated base-band signal is still very fine, as shown in Figure 6b.

In realistic application, co-channel interference is usually inevitable. In order to simulate the multi-path effect

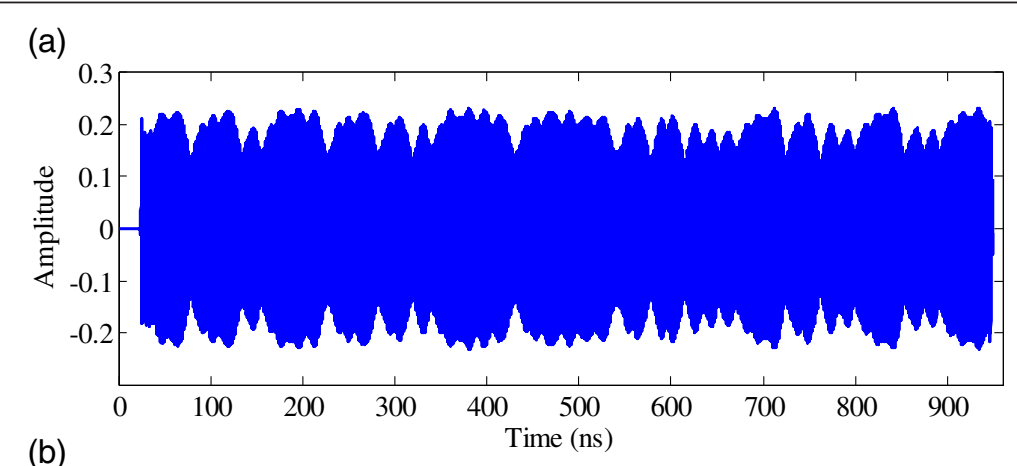

(b)

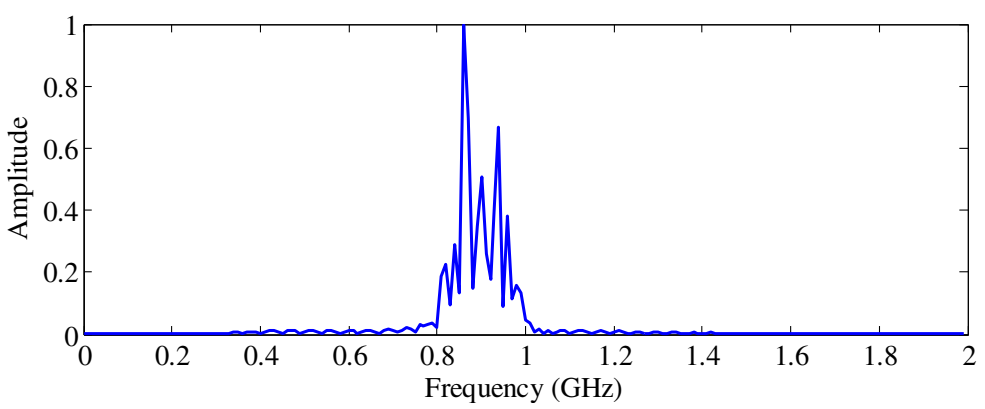

Figure 9 Received signal without spark effect. (a) and (b) show the RF signal received by the dipole without effect of electric spark. 


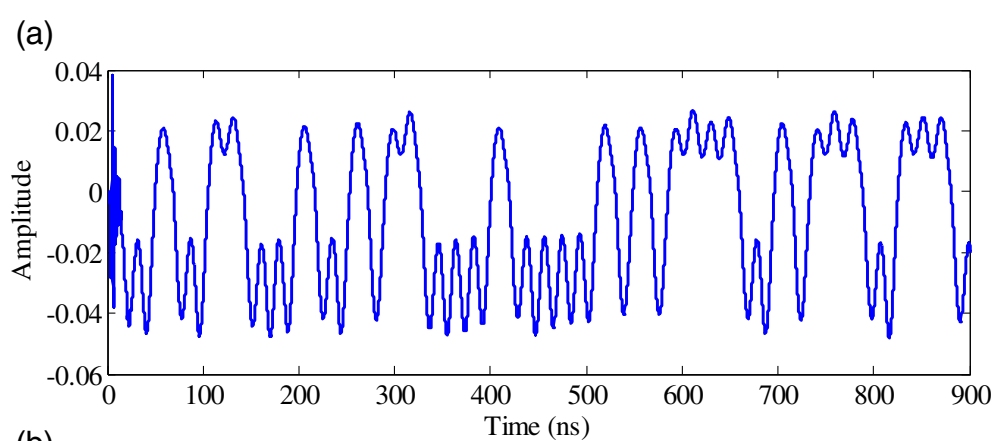

(b)

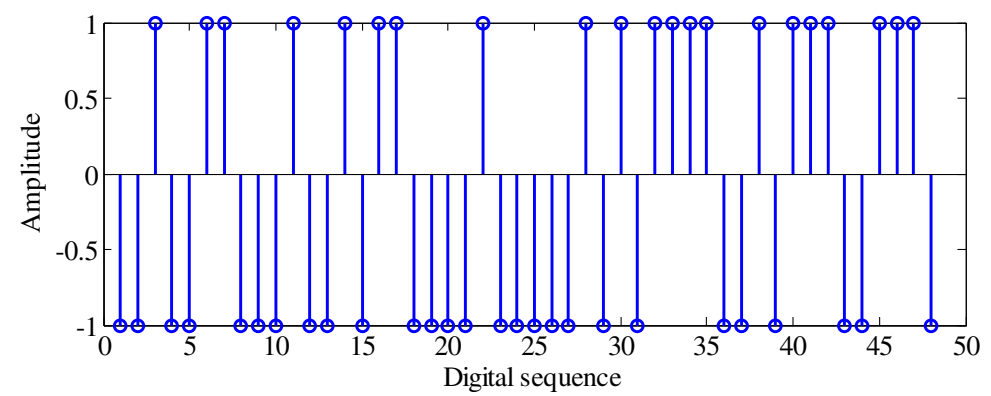

Figure 10 Base-band signal. (a) and (b) show the demodulated base-band and digital signals of system without effect of electric spark.

on the receiving signal, another dipole antenna is introduced in the front of the locomotive with a distance of $9.3 \mathrm{~m}$ to the locomotive antenna. The two dipole antennas are excited by the modulated signals with the same amplitude. The signal received by the locomotive antenna is shown in Figure 7a. Comparing with that in Figure $6 \mathrm{a}$, the waveform of received RF signal is distorted significantly. The demodulated base-band signal is also distorted, as shown in Figure 7b. This will influence the judgment of the digital signal.

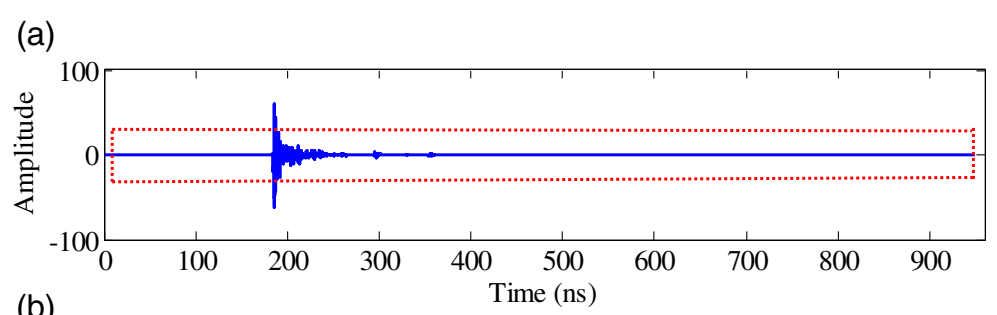

(b)

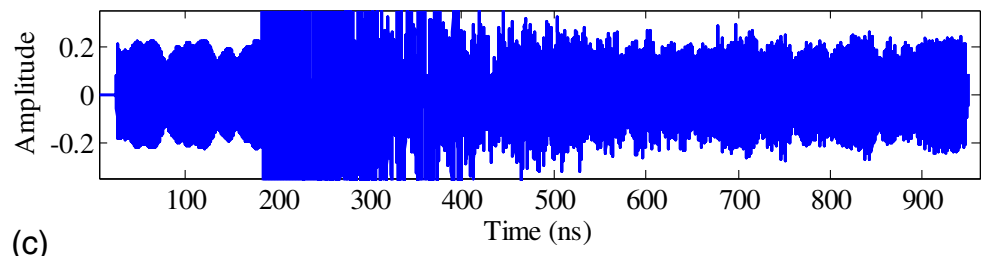

(c)

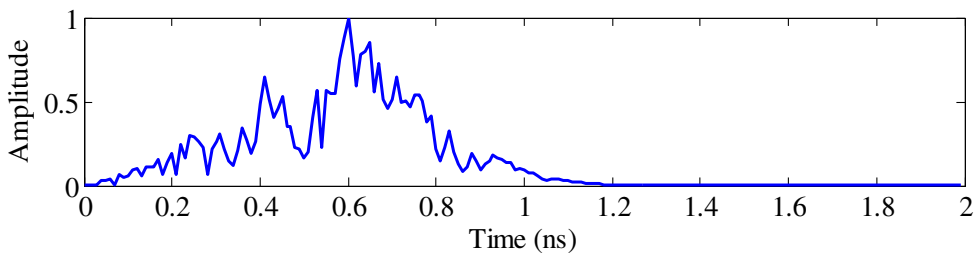

Figure 11 Received signal affected by spark. (a), (b), and (c) show the RF signal and its frequency spectrum which received by the dipole with effect of electric spark. (b) is the enlargement of (a). 
(a)
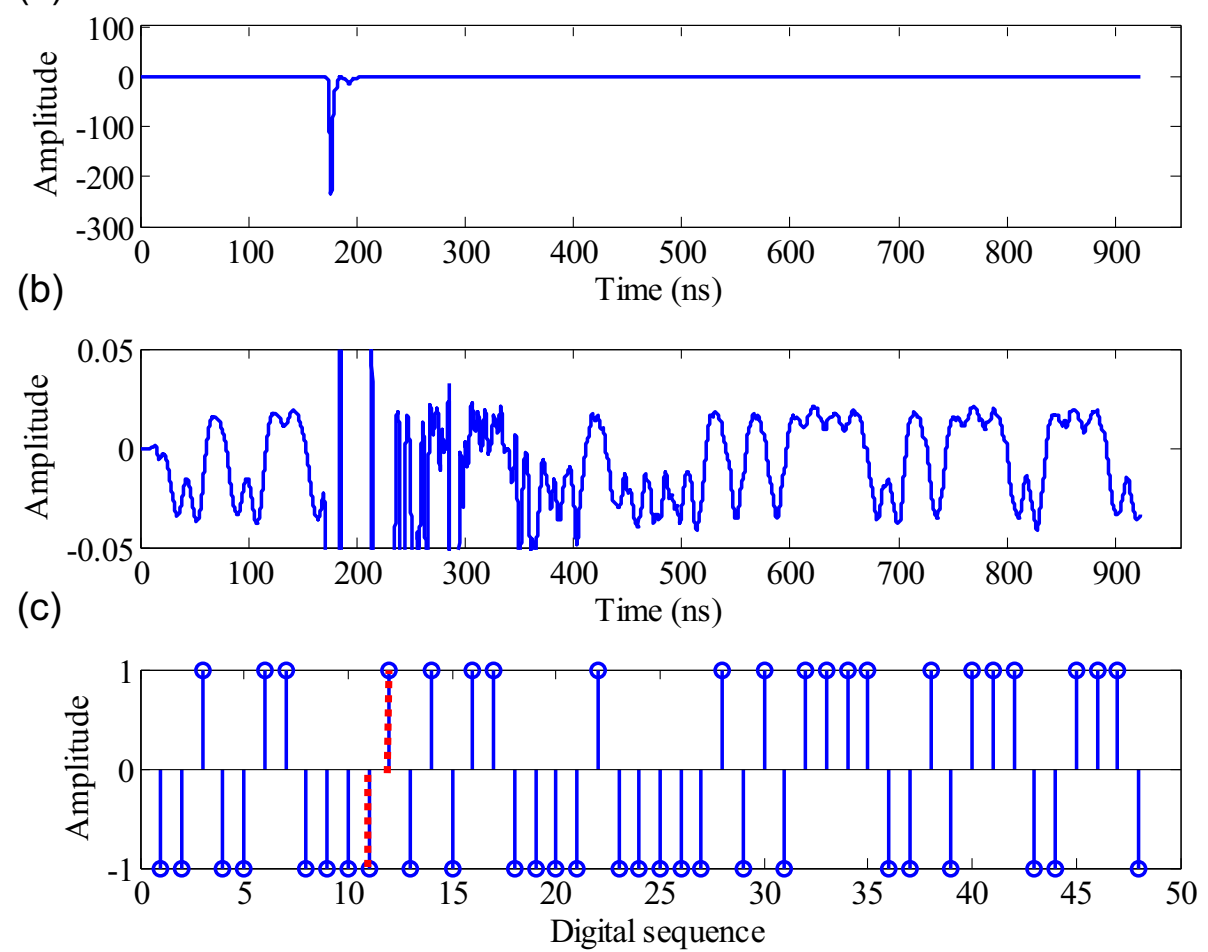

Figure 12 Base-band signal affected by spark. (a), (b), and (c) show the demodulated base-band and digital signals of system with effect of electric spark. (b) is the enlargement of (a).

\subsection{Impact of EMI on base-band digital signal}

It is the normal case for the running train system that the power supply network will generate electric spark. This spark actually is an electromagnetic pulse, which interferes with the transmitting signal. In order to find the impact of this interference on the transmitted digital signal, the generation of the electric spark and its propagation are introduced into the FDTD simulation together with the RF signal. In this example, we let the locomotive antenna transmits and the dipole antenna receives. Assuming that the electric spark has the form of modulated Gaussian pulse:

$$
\begin{aligned}
U_{p}^{n^{\prime}} & =E_{z}^{n} \Delta z \\
& =-U_{0}^{p} \cos \left(2 \pi f_{0}^{p} n^{\prime} \Delta t\right) \exp \left(-4 \pi\left(n^{\prime} \Delta t-t_{0}^{p}\right)^{2} /\left(\tau_{0}^{p}\right)^{2}\right)
\end{aligned}
$$

where $n$ is the iterating time step of FDTD, and $n^{\prime}$ represents the time step at which the spark begin to generate. $U_{0}^{p}$ and $f_{0}^{p}$ are the amplitude and fundamental frequency of the spark, respectively. $t_{0}^{p}$ represents the time moment at which pulse peak occurs. $\tau_{0}^{p}$ is related to the bandwidth, and bandwidth is equal to $2 / \tau_{0}^{p}$. Table 2 gives the values of the parameters.
The digital signal input to the simulation system is shown in Figure 8. The amplitude of the modulated signal is set to $1 \mathrm{~V}$. The FDTD mesh size is $30 \mathrm{~mm}$ which balances the computing time and accuracy. The influence of the electromagnetic spark on the transmitted signal can be analyzed by comparing the receiving signals without and with electrical spark. Figure 9 shows the received RF signal and the corresponding frequency response of the system without spark, and the demodulated base-band signal and recovered digital signal are shown in Figure 10. Comparing Figure $10 \mathrm{~b}$ with Figure 8, we can see that although the RF waveform in Figure 9 seems not perfect, the recovered digital sequence is correct. Figure 11 shows the received RF signal and corresponding frequency response of the system with the effect of the electromagnetic spark, and the demodulated base-band signal and recovered digital signal are shown in Figure 12. From Figure 11, we can see that the transmitted RF signal is submerged in the pulse within the interference period of the spark, and the frequency spectrum of the received RF signal is spread. From Figure 12, we can see that the base-band signal waveform in which period the spark appears is distorted seriously, which further influences the sampling and judgment, and results in bit errors, as shown by the dashed line in Figure 12c. 
It is also found from Figure 12b that the influence of the spark on sampling and judgment lasts more than 200 ns although the spark only lasts 9 ns. This is due to the ringing of the spark pulse on the antenna. In addition, comparing Figure 12c with Figure 8, we can see that the impact of high-intensity electromagnetic pulse on bit error seems not so great (because only two errors occur). However, if comparing base-band waveforms in Figure 12b and Figure 10a carefully, it is found that the judgment of the symbols is at high risk in the period from 200 to $400 \mathrm{~ns}$, because the waveform is significantly distorted. In other words, the accuracy of the symbol judgment cannot be guaranteed between the ninth symbol and the 20th symbol.

\section{Conclusions}

Estimation of the transmission characteristics of wireless link is an important work especially for the wireless communication in complicated environment, such as the high-speed railway environment. But conventional method can only give the field coverage and cannot give the effect of environment on the transmission property of the wireless link. This paper proposed a base-band involved integrative modeling method which can not only take the interaction between antennas and environment into consideration but also give the effect of environment on the base-band signal and bit error rate of the wireless communication. Railway environments are taken as examples to show the implementation and efficiency of the method, and the results show that the proposed method is effective in finding the reason of waveform distortion of base-band signal and can give the explanation of the occurring of bit errors.

\section{Competing interests}

The authors declare that they have no competing interests.

\section{Acknowledgments}

This work was supported in part by NSFC under grant no. 61331002 and in part by the National Key Basic Research Project under grant no.

\section{CB328903.}

Received: 6 June 2014 Accepted: 2 March 2015

Published online: 20 March 2015

\section{References}

1. MF Iskander, Z Yun, Propagation prediction models for wireless communication systems. IEEE Trans. Microw. Theory Tech. 50(3), 662-673 (2006). doi:10.1109/22.989951

2. CF Yang, BC Wu, CJ Ko, A ray-tracing method for modeling indoor wave propagation and penetration. IEEE Trans. Antenna Propag. 46(6), 907-919 (1998). doi:10.1109/8.686780

3. Z Ji, BH Li, HX Wang, HY Chen, Efficient ray-tracing methods for propagation prediction for indoor wireless communications. IEEE Antenna Propag. Magazine 43(2), 41-49 (2001). doi:10.1109/74.924603

4. MS Sarker, AW Reza, K Dimyati, A novel ray-tracing technique for indoor radio signal prediction. J. Electromagnet. Waves Appl. 25(8-9), 1179-1190 (2011). doi:10.1163/156939311795762222

5. K Yee, Numerical solution of initial boundary value problems involving Maxwell's equations in isotropic media. IEEE Trans. Antenna and Propag. 14(3), 302-307 (1966). doi:10.1109/TAP.1966.1138693
6. SD Gedney, An anisotropic perfectly matched layer absorbing medium for the truncation of FDTD lattices. IEEE Trans. Antennas Propag. 44(12), 1630-1639 (1996). doi:10.1109/8.546249

7. G Rodriguez, Y Miyazaki, N Goto, Matrix-based FDTD parallel algorithm for big areas and its applications to high-speed wireless communications. IEEE Trans. Antennas Propag. 54(3), 785-796 (2006). doi:10.1109/TAP.2006.869895

8. AC Austin, MMJ Neve, GB Rowe, RJ Pirkl, Modeling the effects of nearby buildings on inter-floor radio-wave Propagation. IEEE Trans. Antennas Propag. 57(7), 2155-2161 (2009). doi:10.1109/TAP.2009.2021965

9. A Alighanbari, CD Sarris, Rigorous and efficient time-domain modeling of electromagnetic wave propagation and fading statistics in indoor wireless channels. IEEE Trans. Antennas Propag. 55(8), 2373-2381 (2007). doi:10.1109/ TAP.2007.901992

10. M Thiel, K Sarabandi, 3D-wave propagation analysis of indoor wireless channels utilizing hybrid methods. IEEE Trans. Antennas Propag. 57(5), 1539-1546 (2009). doi:10.1109/TAP.2009.2016710

11. M Thiel, K Sarabandi, A hybrid method for indoor wave propagation modeling. IEEE Trans. Antennas Propag. 56(8), 2703-2709 (2008). doi:10.1109/TAP.2008.927548

12. Y Wang, SN Safieddin, SK Chaudhuri, A hybrid technique based on combining ray tracing and FDTD methods for site-specific modeling of indoor radio wave propagation. IEEE Trans. Antennas Propag. 48(5), 743-754 (2000). doi:10.1109/ 8.855493

13. $\mathrm{S} \mathrm{Pu}, J H$ Wang, Z Zhang, Estimation for small-scale fading characteristics of RF wireless link under railway communication environment using integrative modeling technique. Prog. Electromagn. Res. 106, 395-417 (2010). doi:10.2528/ PIER10042806

14. S Pu, J-H Wang, Research on the Receiving and Radiating Characteristics of Antennas on High-Speed Train using Integrative Modeling Technique (Proceedings of 11th Asia Pacific Microwave Conference,(APMC), Singapore, 2009), pp. 1072-1075

15. K Murota, K Hirade, GMSK modulation for digital mobile radio telephony. IEEE Trans. Com. 29(7), 1044-1050 (1981). doi:10.1109/9780470546543.ch20

16. WH Yu, YJ Liu, T Su, N-T Hunag, R Mittra, A robust parallel conformal finite-difference time-domain processing package using the MPI library, antennas and propagation magazine. IEEE. 47(3), 39-59 (2005). doi:10.1109/ MAP.2005.1532540

\section{Submit your manuscript to a SpringerOpen ${ }^{\circ}$ journal and benefit from:}

- Convenient online submission

- Rigorous peer review

- Immediate publication on acceptance

- Open access: articles freely available online

- High visibility within the field

- Retaining the copyright to your article

Submit your next manuscript at springeropen.com 